
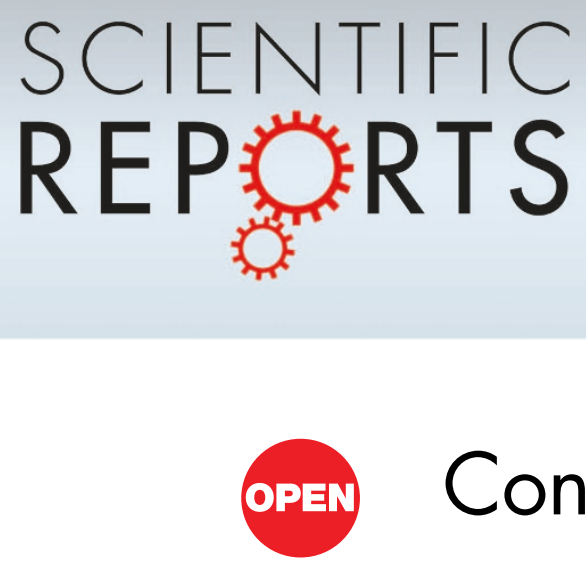

\title{
Controlling avalanche criticality in 2D nano arrays
}

SUBJECT AREAS: ELECTRONIC PROPERTIES AND MATERIALS

CHARACTERIZATION AND ANALYTICAL TECHNIQUES

STATISTICAL PHYSICS

PHASE TRANSITIONS AND CRITICAL PHENOMENA

Received

8 March 2013

Accepted

1 May 2013

Published

15 May 2013

Correspondence and requests for materials should be addressed to Y.P. (paltiel@mail.huji. ac.il)

\author{
Y. C. Zohar' ', S. Yochelis' ${ }^{1}$, K. A. Dahmen², G. Jung ${ }^{3} \&$ Y. Paltiel'
}

\begin{abstract}
'Applied Physics Department and Centre for Nano Science and Nanotechnology, Hebrew University, Jerusalem91904, Israel, ${ }^{2}$ Department of Physics, University of Illinois at Urbana Champaign, Urbana, IL61801-3080, USA, ${ }^{3}$ Department of Physics, Ben-Gurion University of the Negev, P. O. Box 653, 84105 Beer-Sheva, Israel.
\end{abstract}

Many physical systems respond to slowly changing external force through avalanches spanning broad range of sizes. Some systems crackle even without apparent external force, such as bursts of neuronal activity or charge transfer avalanches in 2D molecular layers. Advanced development of theoretical models describing disorder-induced critical phenomena calls for experiments probing the dynamics upon tuneable disorder. Here we show that isomeric structural transitions in 2D organic self-assembled monolayer (SAM) exhibit critical dynamics with experimentally tuneable disorder. The system consists of field effect transistor coupled through SAM to illuminated semiconducting nanocrystals (NCs). Charges photoinduced in NCs are transferred through SAM to the transistor surface and modulate its conductivity. Avalanches of isomeric structural transitions are revealed by measuring the current noise $I(t)$ of the transistor. Accumulated surface traps charges reduce dipole moments of the molecules, decrease their coupling, and thus decrease the critical disorder of the SAM enabling its tuning during experiments.

M any physical systems respond to slowly changing external force through discrete, impulsive avalanches spanning a broad range of sizes ${ }^{1-3}$. A common feature in such systems is that statistics of various avalanche parameters, height, size, or duration, lack a characteristic scale. The far from complete list of systems exhibiting crackling avalanche dynamics includes earthquakes and landslides, Barkhausen noise in ferromagnets, crumpling of paper wraps, acoustic emission in martensitic transformations, fractures propagation, popping foam bubbles, flux penetration into superconductors, and many others ${ }^{1-3}$. There are also systems that crackle without application of apparent external force, such as bursts of neuronal activity in cortex tissue ${ }^{4}$ or charge transfer avalanches in $2 \mathrm{D}$ molecular layers ${ }^{5}$. Advanced development of theoretical models describing disorder-induced critical phenomena calls for experiments probing the dynamics upon tuneable disorder ${ }^{1-3,6}$.

Scale invariance in systems with avalanche dynamics is often attributed to the proximity of a non-equilibrium phase transition ${ }^{1-3}$. Avalanche sizes, $S=\int I(t) d t$ and durations, $T$, in the vicinity of critical disorder $R_{\mathrm{c}}$ have power law distributions $D(S) \propto S^{-\tau}$ and $D(T) \propto T^{-\alpha}$, with universal critical exponents $\tau$ and $\alpha$ depending on the dimensionality and type of criticality of the system. These exponents are related by the critical exponent relation $\alpha-1=(\tau-1) / \sigma v z^{1-3}$. The product $\sigma v z$ determines the exponent of the noise spectral density, which is expected to scale at high frequencies as $S(\omega) \propto \omega^{-1 / \sigma v z}$ and appears also as the power law exponent that characterizes the dependence of the average avalanche size $\left\langle\mathrm{S}>\right.$ on the avalanche duration $\langle S\rangle \propto T^{1 / \sigma v z}$.

Along with exponent relations the most characteristic feature of the critical dynamics is the existence of various scaling collapses involving detail-independent (universal) critical exponents and scaling functions ${ }^{1-3}$.

The detail dependent (non-universal) critical disorder $R_{\mathrm{c}}$ is always determined in terms of the coupling strength in the system $J$. For the $3 \mathrm{D}$ random field Ising model $R_{\mathrm{c}} / J=2.16$. For infinite $2 \mathrm{D}$ systems $R_{\mathrm{c}}=0$, irrespective of the coupling strength. However, space limited 2D systems scale as if critical disorder is greater than zero and the apparent $R_{\mathrm{c}}$ becomes system size dependent ${ }^{6}$. Disorder in the popular random field Ising model is represented by the width of distribution of quenched random magnetic field ${ }^{1-3}$. In strongly ordered systems, $R \ll$ $R_{\mathrm{c}}$, the local field is fairly uniform and a single spin flip can trigger the entire system to flip. With increasing disorder, at $R=R_{\mathrm{c}}$ the spin flip avalanches become scale invariant and cover the entire range of sizes. With disorder increasing slightly above $R_{\mathrm{c}}$ the avalanche size distributions show a disorder dependent cut-off ${ }^{1-3,6}$. However, experimental verifications of effects of changing disorder on the dynamic properties of avalanches are still missing.

In this paper we report on a simple molecular NCs system in which the critical disorder can be in-situ tuned by light illumination. We demonstrate that such disorder changes can be probed by monitoring charge transfer dynamics through the monolayer. 


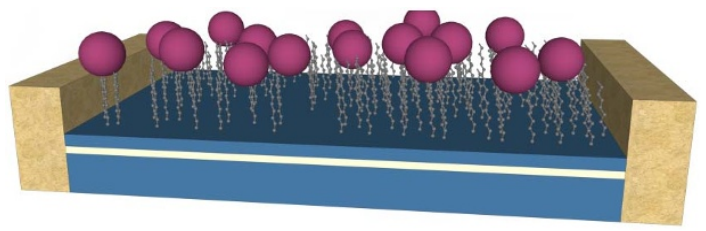

Figure 1 Schematic drawing of the experimental device.

Charge transfer through organic molecular frameworks was extensively studied both theoretically and experimentally ${ }^{7-9}$. Quantum tunnelling dominates conductivity of thiolated alkenes ${ }^{10}$. The tunnelling barrier depends on the type and length of molecules and on their isomeric structure ${ }^{11}$. The barrier for all-trans state is much lower than the barrier for the gauche state ${ }^{12}$. We have recently shown that charge transfer within a molecular layer may have a collective avalanche character associated with disorder driven criticality and nonequilibrium phase transition ${ }^{5}$.

\section{Results}

Schematics of our experimental device are shown in Fig. 1. Colloidal InAs/CdSe/ZnSe nanocrystals (NCs) are covalently absorbed on a self-assembled organic monolayer that links them to the surface of a two dimensional electron gas (2DEG) GaAs/AlGaAs field effect transistor (FET). SAM linker of 1,9 nonanedithiol HS- $\left(\mathrm{CH}_{2}\right)_{9}-\mathrm{SH}$ (NDT), or 1,4 butanedithiol HS- $\left(\mathrm{CH}_{2}\right)_{4}-\mathrm{SH}$ (BDT) molecules is self-deposited from an absolute ethanol solution ${ }^{13}$. The device is
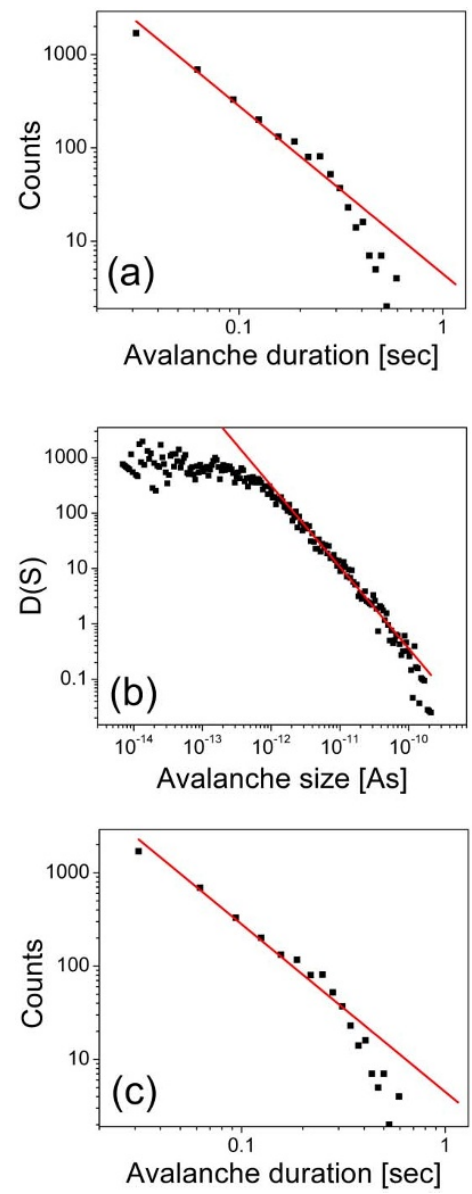

Figure $2 \mid$ Avalanche duration histogram (a), size histogram (b), and the average avalanche size as a function of the duration (c), calculated from the first measurement for NDT device under illumination with a $0.2 \mathrm{~dB}$ attenuator. Solid lines represent best fits to power low distributions. illuminated by light with energies smaller than the GaAs energy band gap and larger than the InAs energy gap. When the NCs absorb light, the photo-excited holes are transferred to the FET surface states via SAM, change the surface potential, bend the conduction band, increase the 2DEG electron density, and thus increase the conductivity of the transistor channel ${ }^{14}$. This has been confirmed by measurements of the DC response of our system to light illumination, showing monotonous decrease of the channel resistivity with illumination time. A change in the FET current can be orders of magnitude larger than the number of photo electrons excited in the $\mathrm{NCs}^{15}$.

Fluctuations in the FET conductivity reflect directly the dynamics of charge transfer through the SAM layer. When collective charge transfer appears, the FET noise is dominated by large non-Gaussian avalanche-like fluctuations. The trigger that initiates an avalanche is a spontaneous transition of a molecular bridge to all-trans configuration. Avalanche propagation is sustained by subsequent isomeric transitions of neighbouring molecules ${ }^{5}$. Negative avalanche pulses correspond to events of charging the surface traps with photo-excited holes, while positive ones correspond to their recombination with electrons in NPs, after the back transfer through the SAM linker ${ }^{5}$.

Figure 2 shows examples of distributions of avalanches size, duration, and average size vs. duration. The distributions follow power laws, suggesting that our system exhibits critical crackling dynamics. This is further confirmed by verification that experimentally determined exponents fulfil the critical exponent relation ${ }^{16} \alpha-1=(\tau-1)$ $/ \sigma v z$ and, most significantly, that individual avalanches of various durations can be collapsed to a universal shape, using the scaling relation $I(T, t)=T^{1 / \sigma v z-1} f_{\text {shape }}(t / T)$, as shown in Fig. 3 . The collapse was obtained with exactly the same $\sigma v z$ exponent as that of the dependence of the average avalanche size on duration.

Avalanches in the investigated system clearly propagate in two dimensions. The values of obtained critical exponents are in extremely good agreement with the exponents obtained by numerical simulations of $2 \mathrm{D}$ random field Ising model ${ }^{6}$. The experimental

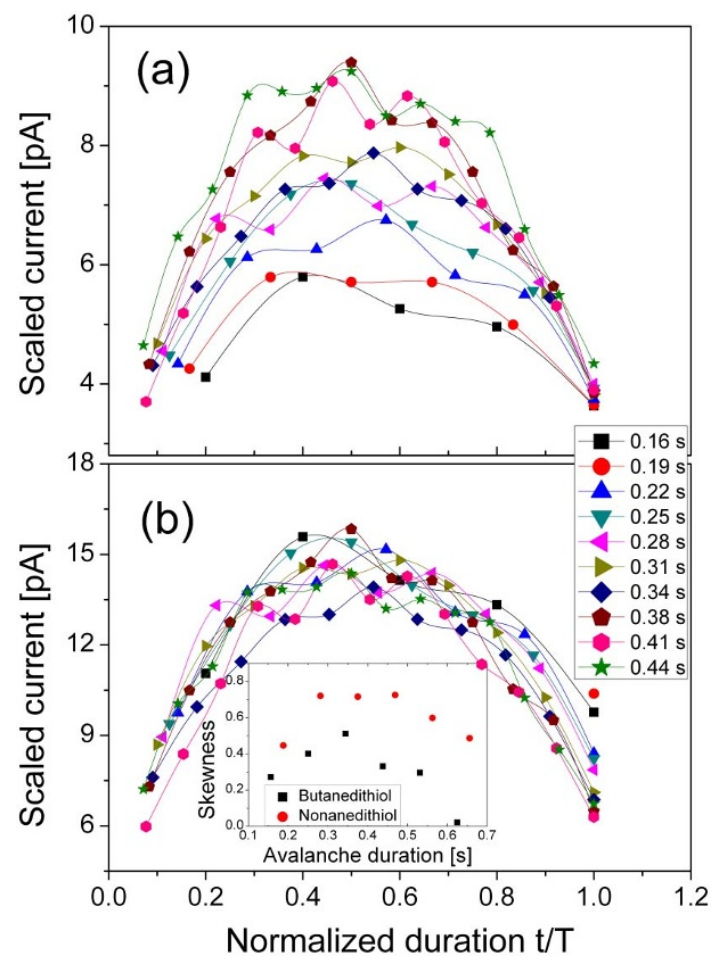

Figure $3 \mid$ Pulse shapes for BDT device before (a) and after (b) scaling collapse. Inset shows the skewness of the pulse shape as a function of the pulse duration. 
exponent $\tau_{\exp }=1.48 \pm 0.12$, while the theoretical $\tau_{\text {th }}=1.5$. The exponent $1 / \sigma v z$ determined from the average avalanche size dependence on duration is $1 / \sigma v z_{\exp }=1.76 \pm 0.26$, the theoretical $1 / \sigma v z_{\text {th }}=$ 1.56 , while the one calculated from the exponents relation is $1 / \sigma v z_{\text {calc }}$ $=1.67 \pm 0.29$. Our results indicate that the molecular system may well belong to the universality class of the random field Ising model $(\mathrm{RFIM})^{1-3}$

The pulse shape predicted by RFIM is a symmetric one. Our experimental collapsed pulse shapes, similar to Barkhausen noise experiments, are clearly asymmetric ${ }^{17,18}$. Pulse asymmetry can be quantified through the pulse skewness $\gamma_{s}=\mu_{3} / \sigma^{3}$, where $\sigma$ is the standard deviation and $\mu_{3}$ the third moment around the mean. As shown in the inset to Fig. 3, our pulse asymmetry is negligible for long and short pulses and peaks for medium length pulses around $350 \mathrm{~ms}$.

Since charges are less likely to tunnel through molecules in the gauche state, the disorder in 2D molecular layer can be associated with the amount of gauche defects in the SAM. In a very clean system the amount of such defects is low. Any avalanche will easily find a path of molecules in all-trans state across the entire system size. At the critical disorder, the avalanches will take all possible sizes and the distribution will fit the power low at all sizes. With disorder increasing above the critical point the percolation of avalanches will be hampered by increasing density of gauche defects, capable of halting the avalanche propagation, and causing the appearance of exponential cutoffs in the power law distribution that eliminate large avalanches.

We have observed that the size and frequency of occurrence of the avalanches decrease with illumination time, to the extent that after some 50-80 minutes avalanches practically disappear. Only by allowing the sample to rest in dark conditions for several hours, the avalanche patterns are fully restored. We ascribe time evolution of avalanches to the loading of FET surface states by holes transferred

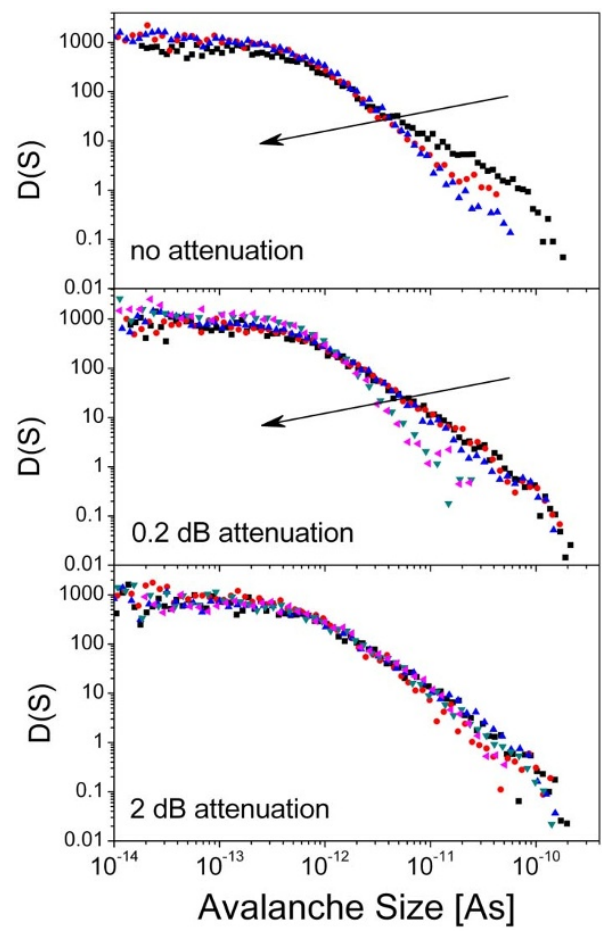

Figure 4 Time evolution of the avalanche size histograms, logarithmically binned and normalized, see the text, at different light intensities; high light intensity (a), intermediate light intensity using $0.2 \mathrm{~dB}$ attenuator (b), and low light intensity using a $2 \mathrm{~dB}$ attenuator (c). Time records were taken at intervals of 16 minutes (0-16 min, 16-32 min, 32-48 min, 48-64 min, 64-80 min). Arrows indicate the direction of elapsed time. from the photo-excited NCs. The probability of avalanche occurrences decreases with increasing fraction of charge loaded surface states. When the amount $N_{\mathrm{C}}$ of charges transferred to the surface is much higher than the amount $N_{\mathrm{T}}$ of available surface traps, as in the case of high intensity light, the charge build-up is governed by surface properties through competition between charge capturing and releasing events. The accumulated charge $Q(t)$ increases as $Q(t) \sim$ $N_{\mathrm{T}}\left\{1-\exp \left(-t / \tau_{\text {eff }}\right)\right\}$, where $\tau_{\text {eff }}$ is the effective rate of charge build up, $1 / \tau_{\text {eff }}=1 / \tau_{\mathrm{c}}+1 / \tau_{\mathrm{r}}, \tau_{\mathrm{c}}$ is the average rate of charge capture and $\tau_{\mathrm{r}}$ the rate of charge release. For very low levels of the illumination power $N_{c} \ll N_{\mathrm{T}}$ and charge build-up becomes controlled by the number of transferred charges $N_{c}, Q(t) \sim N_{c}\left\{1-\exp \left(-t / \tau_{\text {eff }}\right)\right\}$, where $N_{c}(t)=N_{0}\{1-\exp (-\gamma t)\}, \gamma$ depends on the tunnel barrier, and $N_{0}$ is the number of available photo-excited holes in NCs, what makes charging faster for higher level illumination.

Alkene molecules in 2D SAM are coupled through Van der Waals forces associated with the intrinsic molecular electric dipole moment. The vector of the intrinsic moment of dithiol alkens self-assembled on the surface of GaAs points in the direction out of the surface ${ }^{19}$. Therefore, accumulation of photo-excited holes in the surface traps effectively decreases the moment and weakens the coupling. The change in the moment is proportional to the number of charges trapped on the surface. Since $R_{c} / J$ for a given system remains constant, weakened coupling decreases the critical disorder $R_{c}$. Thus, at a given level of disorder in the system $R>R_{\mathrm{c}}$, the distance from the critical disorder will increase exponentially with time as $\left(R-R_{\mathrm{c}}\right) \sim 1$ $-\exp (-\lambda t)$.

Time evolution of avalanche size distributions at different light power levels of NDT molecules linked device is illustrated in Fig. 4. The arrows in the graph indicate the direction of elapsing time. In order to reduce the data scatter in the tails of the distributions we have binned the data using logarithmic binning. Moreover, for purposes of better comparison the distributions have been normalized in such a way that $\int D(s) d s=1$. The major effect of elapsing illumination time is the decrease in the size cut-offs through weakening the coupling $J$ between the molecules.

Theoretical models of disorder induced critical dynamics predict that the cut-offs $S_{\max }$ in the avalanche size distributions should scale as the power law of the distance from the critical point $S_{\max } \sim(R-$ $\left.R_{\mathrm{c}}\right)^{-1 / \sigma 1-3}$. The time dependence of $S_{\max }$ is illustrated in Fig. 5. Since $R$

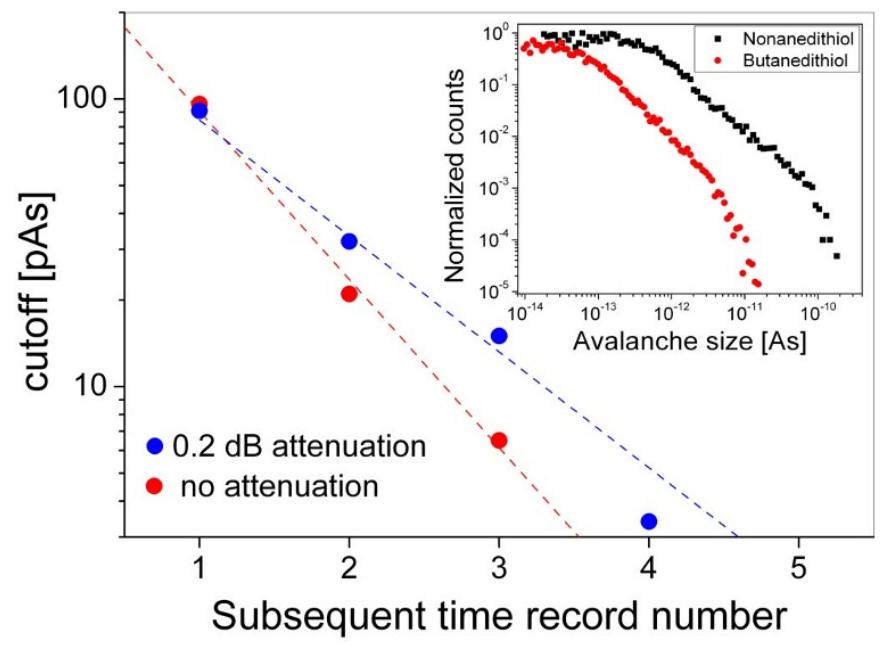

Figure $5 \mid$ Time evolution of the size distribution cut-off $S_{\max }$ as revealed from Fig. 4 for $0.2 \mathrm{~dB}$ and $0 \mathrm{~dB}$ attenuation of the light power. The time axis scale is sequent number of 16 min time record. Dashed lines are the best fits to the logarithmic time dependence of $S_{\max }$. The linear dependence implies that $S_{\max } \sim\left(R-R_{\mathrm{c}}\right)^{-1 / \sigma}$. Inset: size distributions of avalanches in NDT and BDT linked devices under full power illumination. 
$-R_{\mathrm{c}}$ is proportional to $\{1-\exp (-\lambda t)\}$, Fig. 5 actually presents $S_{\max }$ as a function of $\ln \left(R-R_{\mathrm{c}}\right)$. One may conclude that $S_{\max }$ decays as the power law of the distance $R-R_{\mathrm{c}}$ from the critical point. Unfortunately, at this stage we are unable to determine the critical exponent $\sigma$ from the data because we cannot determine the value of the parameter $\lambda$.

Lastly, the coupling between SAM molecules depends also on the molecule length, as testified by decreasing melting temperature of $2 \mathrm{D}$ molecular layers with decreasing molecular length ${ }^{20}$. Our experiments show that indeed $S_{\max }$ for 1,9 nonanedithiol linker is much larger than $S_{\max }$ for shorter and less strongly coupled 1,4 butanedithiol linker, see the inset to Fig. 5.

\section{Discussion}

The growing interest in nonequilibrium phase transitions and advanced development of theoretical models of disorder-induced critical phenomena creates a need for experiments probing crackling noise dynamics in systems with changing disorder. If the model predictions for the connection between the avalanche statistics and the disorder can be proven in experiments, then future noise measurements can be used to extract information about the amount of disorder in a system, without the need for direct measurements of the disorder. These results amount to a new type of nondestructive testing. Most importantly, the predictions for the effects of disorder on the noise statistics are universal, i.e. they are expected to apply to many different systems. This implies that experiments on one of these systems can be used to predict the behavior in many other systems that belong to the same "universality class".

The major body of experimental work concerning disorder induced criticality and associated crackling noise dynamics has been performed using magnetic systems exhibiting Barkhausen noise ${ }^{2}$. However, experimental verifications of effects of changing disorder on dynamic properties of crackling avalanches are still missing. In our paper we discuss dynamics of a hybrid organic-inorganic nano system that exhibits critical avalanche dynamics and show that the critical disorder can be experimentally tuned by light illumination. Our hybrid molecular system enables experimental studies of critical dynamics in which the distance from the critical point depends on density and length of linking molecules and can be tuned during the experiments by changing light intensity and illumination time. The system provides thus the tool box for in-situ studies of critical dynamics under changing disorder. The difference between the coupling of the BDT and NDT molecules leading to different levels of critical disorder in SAMs composed of such molecules present another means of controlled coupling.

Therefore, the investigated hybrid system provides a flexible and controllable tool box for experimental studies of critical dynamics in which the distance from the critical point can be in-situ changed during the experiments. This dynamics is revealed by manifestations of critical charge transfer dynamics, observed through the conductivity noise in the channel of field effect transistor to the surface of which the molecular layer links semiconducting nanocrystals illuminated by infrared light. The critical disorder in the molecular layer can be experimentally tuned by the duration and intensity of light illumination. In addition, the critical disorder can be adjusted by the length and density of molecules in the self-assembled layer, and temperature. The system can be easily fabricated using self-assembly methods. Lastly, our experiments show that charge transfer through alkyl monolayers, frequently used as elements of molecular electronic devices, is a complicated process that can evolve into unwanted noisy avalanche dynamics decimating the performance of practical devices.

\section{Methods}

The FET device. The FET was made by standard lithography techniques from a conducting $\mathrm{n}-\mathrm{GaAs}$ layer, grown on a super-lattice buffer layer of 6 periods ( $\mathrm{Al}$, $\mathrm{Ga}) \mathrm{As}$, each period about $100 \mathrm{~nm}$ thick, and $5 \mathrm{~nm}$ thick insulating layer of GaAs covering the conducting layer. To increase the sensitivity of the device the conducting channel of the FET was shallow and localized between 20 and $50 \mathrm{~nm}$ from the surface SAM linker of 1,9 nonanedithiol HS- $\left(\mathrm{CH}_{2}\right)_{9}$-SH (NDT), or 1,4 butanedithiol HS$\left(\mathrm{CH}_{2}\right)_{4}$-SH (BDT) molecules is self-deposited between the source and drain FET electrodes from an absolute ethanol solution. When the device is illuminated by light, with energies smaller than the GaAs energy band gap and larger than the InAs energy gap, we excite only the NCs, leaving the GaAs parts of the transistor unaffected. When the NCs absorb light, the photo-excited holes are transferred to the FET surface states via the organic molecules, change the surface potential, and thus modify the conductivity of the 2DEG transistor channel. It should be emphasized that FET in our experimental arrangement operates not only as a light detector but also as an amplifier of the light effect.

Nano crystals. We have used in this work InAs $6.5 \mathrm{~nm}$ core and InAs/CdSe/ZnSe core/shell/shell NCs with the same core size. The InAs nanocrystals were synthesized in trioctyl phosphine using InCl3 and TMS3 as precursors and underwent subsequently a size selection process. For the shell growth an ionic layer adsorption and reaction process has been used. The shell consisted of 1 monolayer (ML) of CdSe, followed by $4 \mathrm{ML}$ of $\mathrm{ZnSe}$. As a result, the emission from the NCs at $0.96 \mathrm{eV}$ has improved by more than 2 orders of magnitude.

Measurements. All measurements were performed in a specially designed rod and a cooler sitting inside a Faraday cage. We used a FEMTO DLPCA-200 Variable Gain Low Noise Current Amplifier that was located as close as possible to the rod contacts. The system was situated on a grounded optical table and the rod and amplifier were shielded and grounded to the same ground. Dual channel Stanford research SR785 spectrum analyzer was used to measure the spectra and time response of the system.

1. Sethna, J. P., Dahmen, K. A. \& Myers, C. R. Crackling noise. Nature 410, 242-250 (2001).

2. Sethna, J. P. Crackling Noise and Avalanches: Scaling, Critical Phenomena, and the Renormalization Group. in Complex Systems, Volume LXXXV: Lecture Notes of the Les Houches Summer School 2006. edited by Jean-Philippe Bouchaud, Marc Mezard, and Jean Dalibard, Elsevier, Amsterdam, p. 257 and Xariv:cond-mat/0612418 (2007).

3. Sethna, J. P. et al. Hysteresis and Hierarchies - Dynamics of Disorder-Driven 1stOrder Phase-Transformations. Phys Rev Lett 70, 3347-3350 (1993).

4. Friedman, N. et al. Universal Critical Dynamics in High Resolution Neuronal Avalanche Data. Phys Rev Lett 108, 208102 (2012).

5. Paltiel, Y. et al. Collective Effects in Charge Transfer within a Hybrid OrganicInorganic System. Phys Rev Lett 104, 016804 (2010).

6. Perkovic, O., Dahmen, K. \& Sethna, J. P. Avalanches, Barkhausen Noise, and Plain Old Criticality. Phys Rev Lett 75, 4528-4531 (1995).

7. Anderson, N. A. \& Lian, T. Q. Ultrafast electron transfer at the moleculesemiconductor nanoparticle interface. Annu Rev Phys Chem 56, 491-519 (2005).

8. Hicks, J. F., Zamborini, F. P. \& Murray, R. W. Dynamics of electron transfers between electrodes and monolayers of nanoparticles. J Phys Chem B 106, 7751-7757 (2002).

9. Marcus, R. A. \& Sutin, N. Electron Transfers in Chemistry and Biology. Biochim Biophys Acta 811, 265-322 (1985).

10. Akkerman, H. B. \& de Boer, B. Electrical conduction through single molecules and self-assembled monolayers. J Phys-Condens Mat 20, 013001 (2008).

11. Jorgensen, W. L. \& Gao, J. Cis Trans Energy Difference for the Peptide-Bond in the Gas-Phase and in Aqueous-Solution. J Am Chem Soc 110, 4212-4216 (1988).

12. Paz, Y., Trakhtenberg, S. \& Naaman, R. Phase-Transitions in Organized Organic Thin-Films as Detected by Their Reactivity. J Phys Chem-Us 97, 9075-9077 (1993).

13. Aqua, T., Cohen, H., Vilan, A. \& Naaman, R. Long-range substrate effects on the stability and reactivity of thiolated self-assembled monolayers. J Phys Chem C 111, 16313-16318 (2007).

14. Aqua, T., Naaman, R., Aharoni, A., Banin, U. \& Paltiel, Y. Hybrid nanocrystalsorganic-semiconductor light sensor. Appl Phys Lett 92, 223112 (2008).

15. Paltiel, Y. et al. Uncooled infrared detector using a thin InAsSb layer acting as a gate on a GaAs field-effect transistor. IEEE Sens J 6, 1195-1199 (2006).

16. Mehta, A. P., Mills, A. C., Dahmen, K. A. \& Sethna, J. P. Universal pulse shape scaling function and exponents: Critical test for avalanche models applied to Barkhausen noise. Phys Rev E 65, 046139 (2002).

17. Colaiori, F. Exactly solvable model of avalanches dynamics for Barkhausen crackling noise. Adv Phys 57, 287-359 (2008).

18. Zapperi, S., Castellano, C., Colaiori, F. \& Durin, G. Signature of effective mass in crackling-noise asymmetry. Nat Phys 1, 46-49 (2005).

19. Dubowski, J. J., Voznyy, O. \& Marshall, G. M. Molecular self-assembly and passivation of GaAs (001) with alkanethiol monolayers: A view towards biofunctionalization. Appl Surf Sci 256, 5714-5721 (2010).

20. Schreiber, F. Structure and growth of self-assembling monolayers. Prog Surf Sci 65, 151-256 (2000)

\section{Acknowledgments}

This work has been partially supported by the Israeli Science Foundation, administered by the Israel Academy of Sciences and Humanities (grant 754/09). YP would like to thank the 
Peter Brojde center for innovation. Many thanks to Achim Catalina for throwing such wonderful ideas. Special thanks to Kamil Gradkowski and Uri Banin for providing the nanocrystals. KD thanks the Faculty of Natural Science of Ben Gurion University for hospitality and support through the Dozor program and NSF for support through DMR-1005209.

\section{Author contributions}

Y.C.Z. performed the experiments and initial analysis. S.Y. helped in all aspects related to the chemical process needed to realize the devices. Y.C.Z., Y.P., G.J. and K. A.D. analyzed the data and developed the narrative and major concepts presented in the paper. G.J., Y.C.Z., S.Y., Y.P. and K.A.D. wrote the manuscript.

\section{Additional information}

Supplementary information accompanies this paper at http://www.nature.com/ scientificreports

Competing financial interests: The authors declare no competing financial interests.

License: This work is licensed under a Creative Commons

Attribution-NonCommercial-NoDerivs 3.0 Unported License. To view a copy of this license, visit http://creativecommons.org/licenses/by-nc-nd/3.0/

How to cite this article: Zohar, Y.C., Yochelis, S., Dahmen, K.A., Jung, G. \& Paltiel, Y. Controlling avalanche criticality in 2D nano arrays. Sci. Rep. 3, 1845; DOI:10.1038/ srep01845 (2013). 